\title{
Fear of speaking: chronic anxiety and stammering
}

\author{
Ashley Craig \& Yvonne Tran
}

\begin{abstract}
Stammering results in involuntary disruption of a person's capacity to speak. It begins at an early age and can persist for life for at least $20 \%$ of those stammering at 2 years old. Although the aetiological role of anxiety in stammering has not been determined, evidence is emerging that suggests people who stammer are more chronically and socially anxious than those who do not. This is not surprising, given that the symptoms of stammering can be socially embarrassing and personally frustrating, and have the potential to impede vocational and social growth. Implications for DSM-IV diagnostic criteria for stammering and current treatments of stammering are discussed. We hope that this article will encourage a better understanding of the consequences of living with a speech or fluency disorder as well as motivate the development of treatment protocols that directly target the social fears associated with stammering.
\end{abstract}

Stammering (also called stuttering) is a fluency disorder that results in involuntary disruptions of a person's verbal utterances when, for example, they are speaking or reading aloud (American Psychiatric Association, 1994). The primary symptoms of the disorder are shown in Box 1. If the symptoms are untreated in early childhood, there is a risk that the concomitant behaviours will become more pronounced (Bloodstein, 1995; Craig et al, 2003a).

Although stammering is classified in DSM-IV as a disorder that is typically first diagnosed in childhood (American Psychiatric Association, 1994), it is found in all age-groups. In the latest research on the epidemiology of stammering (Craig et al, 2002) we found that most children who stammer begin to do so before adolescence, most commonly between 2 and 5 years of age, with the highest peak at around 4 years old. This is called developmental stammering. Cases of acquired stammering also occur, where an injury such as stroke or trauma to the brain results in brain damage. We found the population prevalence of stammering over the entire lifespan (from 2 years to older age) to be $0.72 \%$, with at least a $50 \%$ higher prevalence in males. This relatively low population prevalence is to be expected, because although $2-4 \%$ of people develop a stammer in childhood, many recover naturally in early adulthood (Bloodstein, 1995). We found a higher prevalence rate (of up to $1.4 \%$ ) in children and adolescents (2-19 years of age), with males in this

\section{Box 1 Symptoms of stammering}

Behavioural symptoms

- Involuntary repetition of syllables, especially when starting words

- Involuntary prolongation of sounds

- Unintentional blocking on syllables and words, that is, an inability to utter a sound for a brief time, typically 1-3 s

- Unnatural hesitation, interjections, restarted or incomplete phrases and unfinished or broken words

- Concomitant symptoms may include eye blinks, facial grimacing, jerking of the head, arm waving and so on

Psychological and social symptoms

- Emotional struggle when stammering

- Shyness and social avoidance behaviour due to fear of speaking in specific social contexts

- High levels of chronic anxiety, especially social or phobic anxiety

Awarded an honoray doctorate in 2002 for his research and clinical work in neurological disorders, Ashley Craig is now Professor of Behavioural Sciences at the University of Technology, Sydney (Department of Health Sciences, University of Technology, Sydney, New South Wales, Australia 2007. Tel.: +61 29514 1358; fax: +61 29514 1359; e-mail: a.craig@uts.edu.au). He also works as an honorary clinical psychologist at the Royal North Shore Hospital, New South Wales. He has been involved in research into stammering and in its treatment for the past 20 years. Yvonne Tran is currently a senior research fellow at the Key University Centre in Health Technologies, University of Technology, Sydney. Her research work has focused on clinical and neuropsychophysiological aspects of diseases such as spinal cord injury and stammering. 
age-group having a fourfold higher prevalence (Craig et al, 2002).

Over the past 15 years there has been renewed interest in the relationship between stammering and its potential psychiatric consequences such as chronic and social anxiety. The aim of this article is to examine this relationship in the light of latest research and to present appropriate clinical approaches to managing the problem.

\section{Social consequences}

Talking and speaking fluently and effectively to others is a highly valued skill that has many ramifications. Having the ability to speak with fluency and effect will more than likely enhance life opportunities, whereas dysfluency and inarticulacy are likely to confer disadvantage. For example, people with aphasia have been found to have a poorer quality of life (Sorin-Peters, 2003).

The negative social consequences begin early for children who stammer. In primary school they are perceived negatively by their non-stammering peers, may be more susceptible to bullying (Langevin \& Hagler, 2004) and have more difficulty establishing friendships with peers than children who do not stammer (Davis et al, 2002).

Communication fears have been shown to be significantly higher in children who stammer than in children who do not, and these fears increase with age (Hancock et al, 1998). Reduced ability to talk fluently owing to a stammer can limit vocational prospects (Craig \& Calver, 1991; Bloodstein, 1995) and many individuals with a moderate to severe stammer perceive it as a barrier to effective communication and therefore as a negative life event (Craig \& Calver, 1991; Menzies et al, 1999).

Feeling anxious about one's stammer might be considered a reasonable reaction because of its potential to elicit in listeners mockery, embarrassment, frustration or pity (Bloodstein, 1995; Menzies et al, 1999). Consequently, as children who stammer grow into adolescence and adulthood, the risk increases that chronic negative experiences associated with the disorder will precipitate the development of shyness and social avoidance behaviour, limiting opportunities for psychological and educational development (Andrews \& Craig, 1988; Bloodstein, 1995; Craig et al, 2003a). (In defence of people who do not stammer, most will be unaccustomed to the unusual physical and behavioural symptoms associated with the disorder, such as high levels of facial muscle tension, facial grimacing, blocking and frequent repetitions.)

\section{Long-term risks}

If stammering is left untreated, the risk of developing an anxiety disorder in later life may be greater. Longitudinal research following children with speech or language disorders (including stammering) from 5 years of age has consistently found that in early adulthood they have increased rates of anxiety disorders (mostly social phobia) compared with other psychiatric illnesses such as schizophrenia or eating disorder (Beitchman et al, 2001). Negative perceptions and concerns about stammering develop by about 10 years of age (Hancock et al, 1998). Certainly, by adulthood, people who stammer display pervasive negative stereotypes (Craig et al, 2003b). For example, many people with the disorder hold negative perceptions of themselves (such as believing that they are more anxious), and most people who do not stammer, regardless of whether they know someone who does, believe that adults who stammer are shy, self-conscious, anxious individuals who lack confidence (Craig et al, 2003b).

The above research suggests that as one grows older the continued negative effect of a chronic speech disorder such as stammering can be debilitating socially and psychologically.

\section{The experience of stammering and its association with anxiety}

Stammering becomes more severe when an individual is exposed to threatening or demanding stimuli and reduces when the simulus is less threatening. For instance, anxiety-provoking situations such as speaking to an audience or to someone in authority, or to a listener who seems to be impatient or critical, are associated with increased stammering (Bloodstein, 1995). Conversely, the frequency of stammering is generally reduced in situations that are not anxiety-provoking, such as talking to a familiar person or someone not in authority.

Studies have found that, when confronted with threatening social stimuli, people who stammer show physical arousal and report increased anxiety at the moment of stammering (Bloodstein, 1995; Craig et al, 2003a). Heart rate can accelerate just prior to stammering, and when reading aloud people who stammer have been found to have greater heart rate variability, greater respiration changes and greater heart rate acceleration than non-stammering controls (Bloodstein, 1995; Craig et al, 2003a). In people who have a stammer, it has also been found that vasoconstriction is more likely to occur just before stammering than before fluently spoken words (Bloodstein, 1995). 
Levels of state anxiety tend to be higher at the moment of stammering than at non-stammering moments and or in non-stammering controls. For example, Craig (1990) found that people who stammer had substantially higher state anxiety levels than non-stammering controls when talking on the telephone, and Gabel et al (2002) found that people who stammer had higher levels of state anxiety when speaking, as did Peters \& Hulstijn (1984). Ezrati-Vinacour \& Levin (2004) found that severity of stammering correlated significantly with state anxiety during speech tasks, whereas no significant association existed between state anxiety and non-speech tasks.

\section{The association of stammering with chronic anxiety}

The above evidence demonstrates that the risk of stammering increases when an individual with a stammer engages in an anxiety-provoking social interaction, and that the moment of stammering is more likely than not associated with increased levels of physiological arousal and anxiety. However, the extent to which stammering becomes associated with chronic levels of anxiety has remained unclarified. Notwithstanding this, recent research suggests that many people who stammer do present with higher than normal levels of chronic anxiety. Indeed, as mentioned above, some have argued that social fears and anxiety are a reasonable reaction to the symptoms of stammering (Menzies et al, 1999).

Although the available evidence suggests that levels of chronic anxiety in children who stammer are not any different from levels in children who do not (Hancock et al, 1998; Craig et al, 2003a), the picture is different for adults. The majority of studies suggest that adults who stammer are at greater risk of developing chronic anxiety.

In an exhaustive literature search we identified 20 studies that investigated levels of chronic and/ or social anxiety in adults who stammer and nonstammering controls (a full list of these studies and a summary of their results in tabular form are available as a data supplement to the online version of this article, at http://apt.rcpsych.org). Seven studies concluded that adults who stammer were no more anxious than non-stammering controls, whereas 13 concluded that they were more anxious (see online data supplement: Tables 1 and 2). It should be noted that the average number of participants per group in the seven 'no-difference' studies was only 20 , which lessens the confidence one should have in their findings. In the 13 'difference' studies the average was 50 per group.
Design problems are inherent in such studies (Craig et al, 2003a) and a major limitation of almost every study to date is the failure to select a representative sample of people who stammer ('cases'). Most samples consist of individuals who have elected to seek therapy for their stammer, and they are unlikely to be representative (less than $40 \%$ of people who stammer ever seek therapy; Craig et al, 2003a).

To overcome this problem in our own study (Craig et al, 2003a) we used a randomised and stratified design to recruit representative samples of cases and controls from the general population. Over 12000 people were interviewed and people with a stammer were identified using objective assessment of speech as well as corroborating evidence (e.g. that stammering had persisted for more than 3 months). Our findings supported the view that severity of chronic anxiety in people who stammer is significantly higher than in nonstammering controls.

\section{Social anxiety and the diagnosis of stammering}

Social anxiety disorder involves the persistent fear of embarrassment and humiliation, with sufferers avoiding participating in events they think may be potentially distressing, for example public speaking, meetings and social occasions. The disorder is characterised by high levels of generalised anxiety, which can result in severe distress and impede functioning (American Psychiatric Association, 1994).

As discussed above, stammering is characterised by a fear of social events that involve speaking, which can result in embarrassment and distress, as well as by higher than normal levels of chronic anxiety (Craig et al, 2003a). However, DSM-IV criteria forbid the co-diagnosis of social anxiety disorder or phobia in a person diagnosed with stammering (De Carle \& Pato, 1996; Stein et al, 1996). A diagnosis of social anxiety is permitted only in the absence of a disease or condition (such as stammering) that is causing the anxiety (American Psychiatric Association, 1994).

Nevertheless, an individual who stammers may present with social anxiety of a severity similar to that felt by a person with social anxiety disorder, and this anxiety, even though it is probably a consequence of the symptoms of stammering, could be viewed as social phobia (De Carle \& Pato, 1996; Stein et al, 1996; Schneier et al, 1997). Stein et al (1996) found that 12 out of 16 individuals $(75 \%)$ who were seeking therapy for their stammering fitted the DSM-IV criteria for social phobia. However, when 
the diagnosis of social phobia included only those whose anxiety was considered to be in excess of that expected given the severity of their stammering, a sizeable proportion ( 7 of $16 ; 44 \%$ ) still met the DSM-IV criteria for social anxiety disorder. This suggests that the prevalence of social anxiety secondary to stammering might be at least $40 \%$ in people who seek treatment for their stammering. Further research is required to provide accurate data on this prevalence.

Of the 13 studies in our literature search that found significant difference in levels of anxiety between cases and controls, seven investigated social anxiety. All seven showed that people who stammer had higher levels of social anxiety than non-stammering controls (see online data supplement: Table 2). Messenger et al (2004) found that this anxiety was associated with social contexts in which speech tasks might be required but not with contexts such as physical danger. Furthermore, although people with a stammer and people with social phobia present with significantly higher levels of social anxiety than do controls, people with social phobia have been found to have significantly higher levels of anxiety than people who stammer (Mahr \& Torosian, 1999; Kraaimaat et al, 2002).

\section{Discussion and implications for treatment}

As already mentioned, a proportion of people who stammer develop social anxiety of a severity comparable with that of people who receive a primary diagnosis of social anxiety disorder. There is evidence that these individuals can benefit from treatment with therapies suitable for social anxiety disorder (Stein et al, 1996; Schneier et al, 1997). Currently, there is no information regarding differences between people who stammer who do not develop social anxiety and those who do. This remains a research priority.

The best available evidence on the relationship between stammering and anxiety strongly suggests that a rethink of the DSM-IV diagnostic features for stammering is needed. These rightly emphasise symptoms of the dysfluency such as repetitions of syllables, blocking of sounds and so on. However, given that research findings reviewed in this article strongly support the salient role of anxiety (both state and chronic socially based anxiety) in the history of stammering, it seems appropriate that anxiety be considered as an additional diagnostic feature of the disorder, especially in adults. It also seems appropriate to state in the diagnostic features that a proportion of people who stammer present with severe social anxiety.
To inform any changes to the DSM-IV classification, further research is necessary to determine more precisely the nature of the anxiety suffered by people who stammer. Studies using representative samples are needed, and similarities and/or differences between the social anxieties of people with a stammer and of people with social anxiety disorder need investigation. Furthermore, as recognised by others (Stein et al, 1996), the proportion of people with a stammer who also experience levels of social anxiety disproportionate to the severity of their stammering needs clarification.

\section{Management of stammering and related anxiety}

As mentioned above, the evidence we have presented supports the addition of chronic or social anxiety as a diagnostic feature of stammering, especially in adolescents and adults. It is important that DSM-IV diagnostic features for stammering include anxiety symptoms, as their current exclusion can have a detrimental effect on the management of anxiety. For example, a person who seeks treatment for their stammering may not be assessed for their level of related anxiety. They might consequently not be offered appropriate anti-anxiety treatment, simply because anxiety is not viewed as an important component of stammering. Of course, although preliminary evidence suggests that antianxiety treatments can have positive effects for people who stammer (Craig et al, 1996; De Carle \& Pato, 1996; Hancock et al, 1998), further clinical trials are needed to identify aspects of treatment critical to success.

We believe that a more strategic approach to the treatment of stammering is now needed. From the evidence shown here, we might conjecture that as many as $40 \%$ of people who stammer will present with abnormal levels of chronic social anxiety, and that stammering therapies such as fluency shaping and stammering modification techniques (Bloodstein, 1995) that are primarily directed at reducing the stammering behaviour may have limited impact on this anxiety component. An approach to the management of stammering for adolescents and adults is outlined in Box 2. Following detailed assessment, specialised stammering treatment should be provided to help the individual to reduce their stammering by learning control over behaviours such as involuntary repetitions and blocking and overexcessive muscle tension while they struggle to speak. Such therapy is usually conducted within a behavioural therapy regimen (Craig et al, 1996). Fears and anxieties can be indirectly addressed by improving fluency skills as well as reinforcing non-avoidance of situations 


\section{Box 2 The management of stammering in} adolescents and adults

Key components of assessment

- Diagnosis of the severity of stammering in various social contexts

- Diagnosis of the psychological impact of stammering, including social anxiety assessment

- Assessment of social skills repertoire specifically in relation to speech

Treatment

- Specialised behavioural treatment that reduces stammering symptoms by altering speech patterns (e.g. airflow, fluency shaping, speech muscle tension feedback and delayed auditory feedback techniques)

- Stammering modification treatments that can reduce dysfluency by altering stammering responses (including self-control techniques, rewards for fluency and mild punishments for stammering)

- Cognitive-behavioural therapies for social anxieties (e.g. relaxation, social skills and thought-control techniques)

- Pharmacological anti-anxiety therapies for those who have difficulty controlling anxiety using cognitive-behavioural techniques

where the person is at risk of stammering while simultaneously rewarding fluency.

We know that directly treating the stammer can reduce anxiety (Craig, 1990). However, many people who stammer continue to experience high levels of anxiety even after specialised stammering treatment, and relapse (return of stammering) is a risk if the person is anxious (Craig et al, 2003a). People who have difficulties with relapse and anxiety following specific stammering treatments may be those who have a high risk of developing social anxiety. That is, their anxiety has become generalised and associated with many social situations, rather than related specifically to social speaking, when they may suffer some understandable embarrassment about their stammer. For those with persistent social anxiety, anxiety reduction strategies such as cognitivebehavioural therapy and anxiolytic drugs should be considered as an additional primary focus of stammering treatment, perhaps in combination if the anxiety is resistant to the cognitive-behavioural therapy alone. Cognitive therapies can directly target the anxiety by addressing negative attitudes as well as training the individual to be socially assertive and skilled. Relaxation strategies should also be considered. Pharmacological therapy used in addition to the cognitive therapy should be prescribed on an individual basis, for instance if the person has real difficulty in lowering their anxiety in a feared context (e.g. using the telephone). Finally, some people do not want to receive specialised stammering treatment, preferring instead a therapy primarily for their debilitating social anxiety.

We believe that relying solely on treatment that specifically targets stammering behaviour may not be sufficient for the successful management of the disorder, at least for individuals who have abnormally high levels of chronic social anxiety. It is important for the long-term fluency and quality of life of these people that both stammering treatments and anti-anxiety therapies be employed in the management of their dysfluency.

\section{Declaration of interest}

None.

\section{References}

American Psychiatric Association (1994) Diagnostic and Statistical Manual of Mental Disorders (4th edn) (DSM-IV). Washington, DC: APA.

Andrews, G. \& Craig, A. R. (1988) Prediction of outcome after treatment for stuttering. British Journal of Psychiatry, 153, 236240.

Beitchman, J. H., Wilson, B., Johnson, C. J., et al (2001) Fourteenyear follow-up of speech/language impaired and control children: psychiatric outcome. Journal of American Academy of Child and Adolescent Psychiatry, 40, 75-82.

Bloodstein, O. (1995) A Handbook on Stuttering (5th edn). San Diego, CA: Singular Publishing Group.

Craig, A. R. (1990) An investigation into the relationship between anxiety and stuttering. Journal of Speech and Hearing Disorders, 55, 290-294.

Craig, A. R. \& Calver, P. (1991) Following up on treated stutterers. Studies of perceptions of fluency and job status. Journal of Speech Hearing Research, 34, 279-284.

Craig, A., Hancock, K., Chang, E., et al (1996) A controlled trial for stuttering in persons aged 9 to 14 years. Journal of Speech and Hearing Research, 39, 808-826.

Craig, A., Hancock, K., Tran, Y., et al (2002) Epidemiology of stuttering in the community across the entire lifespan. Journal of Speech, Language and Hearing Research, 45, 1097-1105.

Craig, A., Hancock, K., Tran, Y., et al (2003a) Anxiety levels in people who stutter. A randomised population study. Journal of Speech, Language and Hearing Research, 46, 1197-1206.

Craig, A., Tran, Y., \& Craig, M. (2003b) Stereotypes towards stuttering for those who have never had direct contact with people who stutter. A randomized and stratified study. Perceptual Motor Skills, 97, 235-245.

Davis, S., Howell, P. \& Cooke, F. (2002) Sociodynamic relationships between children who stutter and their non-stuttering classmates. Journal of Child Psychology and Psychiatry, 43, 939947.

De Carle, A. J. \& Pato, M. T. (1996) Social phobia and stuttering. American Journal of Psychiatry, 153, 1367-1368.

Ezrati-Vinacour, R. \& Levin, I. (2004) The relationship between anxiety and stuttering: a multidimensional approach. Journal of Fluency Disorders, 29, 135-148.

Gabel, R. M., Colcord, R. D. \& Petrosino, L. (2002) Self-reported anxiety of adults who do and do not stutter. Perceptual and Motor Skills, 94, 775-784.

Hancock, K., Craig, A., Campbell, K. A., et al (1998) Two to six year controlled trial stuttering outcomes for children and 
adolescents. Journal of Speech, Language and Hearing Research, 41, 1242-1252.

Kraaimaat, F., Vanryckeghem, M. \& Van Dam-Baggen, R. (2002) Stuttering and social anxiety. Journal of Fluency Disorders, 27, 319-331.

Langevin, M., \& Hagler, P. (2004) Development of a scale to measure peer attitude toward children who stutter. In EvidenceBased Treatment of Stuttering. Empirical Issues and Clinical Implications (ed. A. K. Bothe), pp. 139-171. Mahwah, NJ: Lawrence Erlbaum Associates.

Mahr, G. C. \& Torosian, T. (1999) Anxiety and social phobia. Journal of Fluency Disorders, 24, 119-126.

Menzies, R. G., Onslow, M. \& Packman, A. (1999) Anxiety and stuttering: exploring a complex relationship. American Journal of Speech-Language Pathology, 8, 3-10.

Messenger, M., Onslow, M., Packman, A., et al (2004) Social anxiety in stuttering: measuring negative social expectancies. Journal of Fluency Disorders, 29, 201-212.

Peters, H. F. M. \& Hulstijn, W. (1984) Stuttering and anxiety. The difference between stutterers and nonstutterers in verbal apprehension and physiologic arousal during the anticipation of speech and non-speech tasks. Journal of Fluency Disorders, 9, 67-84.

Schneier, F. R., Wexler, K. B. \& Liebowitz, M. R. (1997) Social phobia and stuttering. American Journal of Psychiatry, 154, 131.

Sorin-Peters, R. (2003) Viewing couples living with aphasia as adult learners. Implications for promoting quality of life. Aphasiology, 17, 405-416.

Stein, M. B., Baird, A. \& Walker, J. R. (1996) Social phobia in adults with stuttering. American Journal of Psychiatry, 153, 278-280.

\section{MCQs}

\section{Stammering is considered to be:}

a a neurological disorder that results mostly from brain injury during childhood or adulthood

b a childhood developmental disorder that is believed to be inherited and to have a neurological basis

c a psychological disorder caused by being overanxious

d a speech disorder caused by parental upbringing

e a fluency disorder caused by self-esteem problems.
2 Research has shown that:

a anxiety is associated with the moment of stammering

b many people who stammer have high levels of social anxiety

c anxiety worsens the severity of stammering

d treatment that reduces stammering can reduce chronic anxiety levels

e the lifetime population prevalence of stammering is the same for males and females.

3 Generally, treatment for stammering should focus primarily on:

a reducing stammering symptoms and the socially based anxiety linked to speaking

$\mathrm{b}$ reducing stammering alone

c resolving family dynamics

$\mathrm{d}$ reducing chronic anxiety alone

e reducing fears at the moment of stammering.

4 Stammering is best diagnosed by:

a measuring social anxiety levels in children using validated questionnaires

b measuring family interactions with the stammering person

c measuring the stammering behaviour and associated anxiety levels

d measuring levels of self-esteem in children

e measuring brain activity levels using electroencephalograms.

5 Stammering is classified as:

a a developmental speech disorder

$\mathrm{b}$ an anxiety disorder

c a neurological deficit disorder

$\mathrm{d}$ a conduct disorder

e a learning disorder. 